\title{
Estrategias didácticas en el desarrollo del pensamiento crítico en estudiantes de educación básica*
}

\author{
Didactic strategies in the development of critical thinking \\ in secondary school students
}

\author{
LUIS Alberto NúNÉz-LIRA** \\ Dally Mayer Gallardo-Lucas*** \\ Alicia Agromelis Alaga-Pacore**** \\ Jorge Rafael Diaz-DUMONT*****
}

\section{Resumen}

Objetivo. Evaluar las estrategias didácticas usadas en las sesiones de aprendizaje del área de comunicación para desarrollar el pensamiento crítico en estudiantes de educación secundaria de instituciones educativas de la jurisdicción de Ocros. El desarrollo del aprendizaje en el siglo XXI exige que sus estudiantes desarrollen altos niveles de pensamiento y creatividad, utilizando diversas estrategias pedagógicas por parte de la comunidad docente. Metodología. Se utilizaron los diseños cuantitativo y cualitativo, por lo tanto, este estudio se caracteriza por ser de naturaleza mixta; triangulándose no solo datos, eventos y entrevistas aisladas, sino también el análisis y la reflexión pedagógica. Se utilizaron los diseños cuantitativo y cualitativo, los cuales permiten analizar e inferir cómo los docentes de la provincia de Ocros-Huaraz-Perú desarrollan la gestión del aula. Resultados y conclusión. El pensamiento crítico, en sus tres subcategorías: razonamiento, argumentación y toma de decisiones, permite afirmar que los docentes presentan fuertes debilidades en su proceso de conducción de los aprendizajes y los estudiantes carecen de herramientas cognitivas que posibiliten realizar una lectura crítica.

Palabras clave: pensamiento crítico, estrategias didácticas, razonamiento, argumentación, toma de decisiones.

\begin{abstract}
Objective: To evaluate the didactic strategies used in the learning sessions of the communication area to develop critical thinking in secondary school students of educational institutions in the jurisdiction of Ocros. The development of learning in the 21 st century requires that students develop high levels of thought and creativity, using various pedagogical strategies by the teaching community. Methodology: Quantitative and qualitative designs were used so this study is characterized as being of a mixed nature, triangulating not only data, events and interviews, but also the pedagogical analysis and reflection. Quantitative and qualitative designs were used which allow analyzing and inferring how teachers in the province of Ocros-Huaraz-Peru develop classroom management. Results and conclusion: Critical thinking, in its three subcategories, reasoning, argumentation and decision-making, allows affirming that teachers have strong weaknesses in their process of conducting learning, and students lack cognitive tools that make it possible to carry out critical reading.
\end{abstract}

Key words: critical thinking, didactic strategies, reasoning, argumentation, decision-making.

\footnotetext{
* La investigación dio como resultado que docentes y estudiantes no han desarrollado las competencias requeridas. Los docentes presentan limitadas capacidades pedagógicas y los estudiantes no han desarrollado las herramientas cognitivas que les permitan desarrollar el pensamiento crítico.

${ }^{* *}$ Universidad César Vallejo. Lima, Perú. E-mail: lnunezl@ucv.edu.pe. (1) orcid.org/0000-0003-3542-9117 Google Scholar

*** Universidad César Vallejo. Lima, Perú. E-mail: dallyta04@gmail.com (10 orcid.org/0000-0001-5672-1948

***** Universidad Nacional Federico Villareal. Lima, Perú. E-mail: aaliagap@unfv.edu.pe. (1) orcid.org/0000-0002-4608-2975

****** Universidad Nacional Autónoma deTayacaja Daniel Hernández Morillo. Huancavelica, Perú. E-mail: jorge.diazdu@ciplima.org.pe (D) orcid.org/000-0003-0921-338X Google Scholar
} 
Estrategias didácticas en el desarrollo del pensamiento crítico en estudiantes de educación básica

\section{Introducción}

La educación en todos los sistemas educativos tiene como propósito desarrollar las capacidades que posee la persona (Sanz y Serrano, 2017), para lo cual, a través de los programas curriculares, pone en marcha un conjunto de actividades pedagógicas que contribuyan al desarrollo del lenguaje, pensamiento, reflexión, creatividad, autonomía, emprendimiento y demás facultades humanas. Las capacidades humanas integran los saberes, el afecto, la socialización y los principios de vida. Estos aspectos se logran de modo procesal y, si se orienta desde la familia, la sociedad y la educación básica, entonces se tendrá estudiantes capaces de desempeñarse en contextos diversos a los que se desenvuelve (Tobón, 2013).

Así, las instituciones educativas (II. EE., en adelante) hoy tienen la responsabilidad de formar personas capaces de aprender en un contexto favorable y de forma sistemática, donde el estudiante aprenda poniendo en práctica la teoría, indague, cuestione y reflexione. Es decir, que el aprendizaje en la educación básica le sirva al estudiantado para afrontar situaciones diversas en sus experiencias de vida y en los contextos donde se desenvuelva. En esta línea la educación debe centrarse en la persona y en la interrelación con su contexto para buscar respuestas a los problemas humanos con relación al comportamiento, a los hechos sociales y hacia dónde vamos como sociedad (Morin, 1999).

Con el fin de mejorar la práctica pedagógica docente, los órganos intermedios de Educación en la Provincia de Ocros - Ancash monitorean y acompañan la labor pedagógica de manera progresiva, usando un instrumento denominado Ficha de Observación de Aula (2019). Mediante este, se recoge información referida a desempeños observables explicitados en el Marco del Buen Desempeño Docente. Finalizada la observación de aula, se toma un tiempo para el acompañamiento docente, destacando ahí los aspectos positivos para fortalecer, potenciar o señalar los aspectos que requieren atención para obtener resultados educativos favorables.

El propósito en el área de comunicación es generar en los estudiantes aprendizajes que les permitan argumentar que, a través del establecimiento de un conjunto de premisas, puedan llegar a conclusiones. En este proceso el estudiante debe aprender a usar los referentes teóricos como fundamento para los argumentos; también debe lograr analizar y sintetizar las ideas de un texto escrito u oral para hallar la relación lógica entre las ideas del texto (Castelló, Clariana, Monereo, Palma y Pérez, 2000).

En nuestro país, desde 2015 se aplican evaluaciones censales a estudiantes de segundo grado de secundaria cuyos resultados se expresan en niveles de logro. Así, en el 2018 los resultados indicaron que en el nivel previo al inicio se ubica el 18,5\%, en inicio 37,5\%, en proceso, $27,7 \%$ y en satisfactorio, $16,2 \%$. 
Entonces, es de nuestro interés indagar cómo durante el proceso pedagógico de la enseñanza y aprendizaje del área de comunicación, el docente debe estimular, promover o generar el pensamiento crítico, qué técnicas o estrategias usa para el desarrollo, en qué momento del proceso didáctico promueve esta capacidad de pensamiento de orden superior o en su defecto, qué tipo de actividades de aprendizaje se desarrollan oralmente para estimular la capacidad crítica en las II. EE. del nivel secundaria. La formulación del problema plantea: ¿Qué estrategias didácticas se usan en sesiones de aprendizaje del área de comunicación para desarrollar el pensamiento crítico en estudiantes de educación secundaria de las II. EE. educativas de la jurisdicción de la UGEL Ocros - Huaraz?

En referencia a los procesos didácticos, el hecho educativo se concretiza en el proceso de enseñanza y aprendizaje. Por lo tanto, el segundo cumple un rol preponderante que se da en la interacción estudiantil, donde cada uno tiene una cultura propia pero el interés común de aprender. El docente, para la enseñanza, ha de crear condiciones adecuadas y media en el proceso didáctico (Espinoza, 2016), mediante la proporción de materiales y el diseño de estrategias que contribuyan a movilizar las diversas capacidades y, en particular, lo referente al pensamiento crítico.

En el aprendizaje se evidencia la combinación de capacidades diversas, en situaciones y contextos diferentes (Feldman, 2010). Por ello, el docente ha de cumplir una labor eficaz que evite el retraso educativo mediante el dominio disciplinar, el manejo de estrategias y métodos didácticos según los propósitos de enseñanza y aprendizaje (Pimienta, 2012); y las características del estudiantado respecto a cómo aprende, qué aprendizajes posee, qué factores pueden favorecer su aprendizaje en el contexto donde se desarrolla.

Vista la enseñanza, incluye tácitamente los procesos de mediación para el desarrollo de los aprendizajes que integran estudiantes, docentes y la disciplina científica que, para ser aprendida, requiere de la experiencia del pedagogo, de los materiales y las estrategias didácticas (Espinoza, 2016). Además, incluir aspectos que impacten en la subjetividad del estudiantado, generando motivación o emociones que le permitan estar predispuesto a lograr los aprendizajes planificados y desarrollar capacidades y habilidades.

El momento que comprende la enseñanza y el aprendizaje debe entenderse como el fundamento para la formación integral de la persona, destacando su desarrollo para el aprendizaje continuo y favoreciendo las capacidades de orden superior, como es el pensamiento crítico. En una clase debe asegurarse que todos aprendan a observar, analizar, interpretar, emitir una opinión o argumentar posturas propias en el marco de bases teóricas. Este propósito educativo debe ser asumido en todos los niveles educativos, y mejor aún, durante la educación básica. Así también ha de ser transversal en todas las líneas curriculares (Chrobak, 2018; Suárez, Colón, Cohen y Colpas, 2017). 
Estrategias didácticas en el desarrollo del pensamiento crítico en estudiantes de educación básica

¿Pero cuáles son los medios didácticos para favorecer que los estudiantes sean observadores, que razonen, interpreten y logren una idea, opinión o argumento? Primero, es la interrelación entre pares y con el docente, seguido de la reflexión intrapersonal, continuando, el docente y su rol mediador por el que promueva actividades académicas intencionales que contribuyan a generar los procesos cognitivos señalados, con soporte de estrategias y procedimientos específicos.

Este parece ser un tema nuevo; sin embargo, la acción humana nos conduce a ver que como sociedad todavía no nos desenvolvemos con responsabilidad y autonomía, por lo cual, en el sistema educativo peruano, el enfoque por competencias, entre los diferentes aspectos del desarrollo del estudiante, pone atención en el logro del razonamiento, la creatividad y el pensamiento crítico (Rodríguez y Pinto, 2018; Ministerio de Educación, 2018).

La gestión escolar garantiza este proceso a través de las acciones de monitoreo y acompañamiento. Conocidos los resultados de desempeño pedagógico, el directivo, los presenta a la plana docente para la toma de decisiones conjunta buscando que las acciones pedagógicas incidan en el progreso del aprendizaje, siendo este el reto educacional. Para ello, es necesario laborar en torno a una visión institucional, seguir los planes establecidos, analizar por tramos los resultados y corregir o fortalecer las acciones centradas en el desarrollo de competencias, como parte de la formación integral de la persona (Muñoz, 2019; Tobón, 2013).

La formación de la persona no solo implica centrarse en el desarrollo cognitivo, cuando la mirada que se tiene de la persona es holística. La persona no solo aprende el "saber conocer", sino también, "saber ser, saber convivir y saber hacer" (Tobón, 2013).

Los estudiantes llegan a las aulas con el interés de aprender; sin embargo, sostener y potenciar esto requiere de permanente motivación tanto interna como externa. Esta última delineará acciones heterogéneas para desarrollar la creatividad, la indagación, el descubrimiento, la innovación, la búsqueda de respuestas a problemas diversos. Por ende, la búsqueda de resultados educativos conduce a avizorar acciones basadas en propuestas o estrategias que generen interés y sentido frente a lo que se aprende (Gregorzewski, 2018; Magen-Nagar y Cohen, 2017).

Ejercer la docencia exige ser profesionales con dominio del campo disciplinar, con experiencia en la investigación científica para la búsqueda constante de respuestas a la diversidad de problemas educativos y el dominio de la pedagogía, basado en una relación horizontal con el discente. El docente en su rol pedagógico orienta el desarrollo de la capacidad reflexiva sobre la realidad social, de tal forma que los aprendizajes no queden en una esfera abstracta, sino arriben en el análisis de la realidad para asumir un pensamiento propio (Júlia \& Formosinho, 2018; Drovnikov, Vazieva, Khakimova y Álvarez, 2018). 
El éxito del proceso pedagógico se centra en la didáctica, la cual debe implementarse con estrategias diversas evitando solo la pedagogía directiva y estimular el aprendizaje por diversos medios. El docente, sujeto del proceso de formación continua, buscará otros escenarios activos e interactivos, implementándolos para que el estudiantado inmerso en un mundo dinámico aprenda según sus caracteres, expectativas e intereses. Por lo tanto, si la mirada es formar estudiantes reflexivos, esta debe empezar por los educadores, quienes deben reflexionar sobre cómo pueden mejorar su didáctica (Popescu y Chisega, 2017; Maccario, 2017).

Dejar a la voluntad e interés del estudiante su formación integral puede poner en peligro, no solo el aprendizaje sino también sus actitudes y valores dentro y fuera del espacio escolar (Vences \& Antony, 2017; Kalin, Peklaj, Pečjak, Levpušček \& Zuljan, 2017; Spoden \& Fricke, 2018; Lanzat, Gil, González, Cacheiro y Pérez, 2018).

El propósito de brindar educación debe asumirse con responsabilidad en los diversos espacios sociales, con la labor de los actores educativos que contribuya a tener una sociedad que destaque por poseer una actitud pensante, reflexiva y sensata. Así de relevante, es también, el soporte emocional, factor indispensable para el aprendizaje y la integración social (Ministerio de Educación, 2003; Díaz, Vázquez y Díaz, 2019).

La acción educativa, según el enfoque por competencias, se alcanza cuando los estudiantes usan su aprendizaje en una situación determinada, con un fin específico, actuando en correspondencia con las normas y la capacidad reflexiva. Para tal fin, el docente, luego de generar las condiciones socio-emocionales, desarrollará una enseñanza activa e interactiva en los procesos pedagógicos, mediante acciones, medios y materiales diversos que asegure el aprendizaje, con miras, al logro esperado (Ministerio de Educación, 2019; Tobón, 2013).

En referencia al pensamiento crítico, este tipo de pensamiento favorece el razonamiento inductivo o deductivo a partir de premisas propuestas o de la realidad. La reflexión, aun cuando es una facultad humana, se aprende en el proceso de la vida, con apoyo de métodos, estrategias o materiales que evitan la sola orientación teórica. Sin embargo, en el contexto escolar, se observa con preocupación la poca reflexión sobre los diferentes sucesos que se vive, lo cual proyecta, la imagen de un grupo humano con poca iniciativa para transformar su realidad. Entonces, el razonamiento o la reflexión son capacidades que manifiestan el cuestionamiento frente a los estímulos recibidos y procesa, como lo refieren Arias (2018), Standish y Thoilliez (2018) y Capinzaiki, Santana y Moreira (2016).

El desarrollo de la criticidad es una responsabilidad que la educación debe asumir de modo transversal, porque permite que la sociedad se desenvuelva pensando antes de actuar y actuando con base en un conocimiento. También, ser personas críticas nos hace actuar respetando las ideas de otros, así como las razones que sustentan sus acciones en el marco de los valores y la 
Estrategias didácticas en el desarrollo del pensamiento crítico en estudiantes de educación básica

ética. No obstante, se observa que hay actos que nos hacen pensar en el poco trabajo educativo por orientar acciones educativas que formen personas reflexivas.

Estas acciones las podríamos definir: el poder político, económico, social, educativo que manipula y se aprovecha de la confianza de quienes no analizan ni deciden para favorecer a aquel que tiene poder de dominio. ¿Pero en qué momento de la vida se aprende a ser críticos? Desde que somos vida, somos seres pensantes y su desarrollo está en la influencia de la institución educativa (Habermas, 1999; Madrid, 2018; Salica, 2018).

La capacidad crítica se enseña y aprende en contextos reales o simulados de modo activo, apoyado en el uso de materiales iconográficos, textuales y audiovisuales que generen desequilibrio cognitivo en el contexto del "debe ser". Es decir, estar frente a situaciones problemáticas, debe conducir a emitir opiniones para presentar ideas o propuestas que conlleven una solución para transformación y el desarrollo del "no debe ser". De ser así, la educación, cumplirá el propósito delineado en el Currículo Nacional de la Educación Básica, que es formar estudiantes para desenvolverse con competencia en los ámbitos intrínsecos y extrínsecos (Grez, 2018; Chrobak, 2017; Kusumoto, 2018).

La competencia lectora, según los niveles que evidencia su logro, exige que el lector reflexione, evalúe el texto, y emita una opinión justificada. Cuando una persona alcanza esta formación, puede decirse que no solo está preparada para afrontar situaciones en el ámbito escolar o académico, sino en la vida misma, en lo personal y social (Avendaño, 2016).

Mejor desarrollo de esta capacidad lo tendrán quienes reciben estimulación desde la infancia, con orientaciones desde la propia experiencia de vida. Caso contrario quien actúa críticamente, pues siempre estará buscando formas nuevas de actuar para responder creativamente a las diversas situaciones que deba enfrentar.

Frente a las ideas vertidas, urge que las instituciones educativas pongan atención en desarrollar procesos cognitivos que favorezcan la capacidad de razonar, para lo cual, los estudiantes propongan soluciones a una diversidad de hechos o asuntos que exijan ser resueltos. Para ello, necesita usar estrategias que le conduzcan a hacer deducciones o conjeturas que luego, le permitan arribar a conclusiones y propuestas fundamentadas en la razón (Siegel, 2010).

El estudio desarrollado por Medina (2017) presenta un conjunto de pasos para asegurar el aprendizaje, tomando como aspecto central en la didáctica docente. Entonces, la planificación para el aprendizaje ha de comprender: a) la generación de un contexto motivante, b) la presentación de situaciones desafiantes, susceptibles de ser comprendidas y analizadas, las cuales correspondan con los conocimientos previos, c) orientaciones para el análisis de las situaciones desafiantes y propuestas de posibles soluciones, d) orientaciones para el uso de 
conceptos y categorías lingüísticas que permitan argumentar, deducir, interpretar, y llegar a conclusiones, e) orientaciones para la toma de decisiones frente a las soluciones propuestas (Vygotski, 2009).

Las estrategias para movilizar la capacidad de razonamiento en estudiantes de educación secundaria son: a) formulación de proposiciones, b) análisis de las proposiciones en el contexto del texto, c) integración de las proposiciones, d) comprensión del sentido del texto a través de las anáforas, e) comprensión del sentido del texto a través de los conectores, f) análisis de adverbios de enmarcamiento (de lugar y distribución del texto), g) conexión de ideas explícitas e implícitas, h) emisión de respuestas a preguntas sobre el propósito del texto y su sentido global de esta emisión de las conclusiones del texto (Aguilera y Andueza, 2018; Bloom, 1974).

Las estrategias que contribuyen al desarrollo de la capacidad argumentativa son: a) conocer un tema o asunto a tratar sea o no como parte de un texto de lectura, b) el planteamiento de problemas frente al texto de lectura o asunto en general, b) la emisión de opiniones o argumentos frente al planteamiento de problemas presentados como propuestas o posibles soluciones, c) la búsqueda de información para justificar la opinión u argumento, d) la justificación de las opiniones o argumentos como respuesta a las interrogantes, y e) la contrastación o análisis de los argumentos u opiniones según los sustentos teóricos (Toulmin, 2003; Atienza, 2005).

Saber tomar decisiones es una capacidad afín al razonamiento y a la argumentación. Por lo tanto, quien posee las capacidades señaladas, han de usar estrategias para la toma de decisiones a partir de: a) la identificación de hechos que denotan problema, b) la identificación de medios para la solución, c) la determinación de medios para la solución, d) el análisis de los medios de solución e) la jerarquización de los medios de solución, f) la selección de los medios de solución (Rodríguez y Pinto, 2018).

En referencia a las teorías, enfoques conceptuales donde se enmarca la investigación, nos preguntamos si el pensamiento crítico es una capacidad que se desarrolla de modo nato o en una relación de enseñanza y aprendizaje. Según afirmaciones hechas por Ennis (2011), los aprendizajes se concretizan en las clases, a través del desempeño pedagógico docente, pues los estudiantes aprenden interactuando con sus pares, movilizando el razonamiento, la imaginación, la invención, la creatividad, la expresión oral, el juicio y la reflexión crítica.

En el periodo de la infancia se evidencia la capacidad inquisitiva del niño, quien todo lo que abstrae, lo interroga. Durante la segunda infancia (2 a 7 años), el niño otorga vida a todas las cosas al querer conocer la información que ingresa por sus sentidos, cuestiona; pero no lo hace desde la búsqueda de razón o desde la causalidad de un hecho, sino desde la naturaleza de lo que le inquieta, como lo refiere Piaget (1991). La enseñanza y el aprendizaje para pensar críticamente, si se desarrollan durante la educación básica, tendrán repercusión en la 
vida adulta, porque cuando más temprano se aprende a actuar podrá adquirirse autonomía, confianza, capacidad de decisión y valores (Elder, 2003).

¿Pero qué es pensar críticamente? Según Elder y Paul (2005) es gestionar con autonomía el propio conocimiento o la cultura universal, los cuales son dinámicos en el tiempo y contexto. Saber pensar críticamente es llegar a analizar y evaluar aquello que es susceptible de modificación, antes de seguir repitiendo de modo explícito la realidad. En el proceso de análisis y evaluación se dinamizan los aspectos fundamentales de la capacidad crítica, como la imaginación, que es un aspecto fundamental para la criticidad.

El pensamiento se desarrolla progresivamente desde la infancia, según se va adquiriendo el lenguaje. Por lo tanto, como Piaget (1991) señala, ambas capacidades son inherentes entre sí. Esto demanda que la educación considere estrategias para contribuir a que el estudiante se empodere del lenguaje y del sistema de signos lingüísticos en la socialización con los de su entorno. En esta línea de ideas, una actividad pedagógica que incide en aspectos referidos al pensamiento crítico es la lectura crítica, pues genera espacios para que los estudiantes dialoguen, opinen, cuestionen y sustenten su posición.

La competencia pedagógica del docente, así como los procesos de mediación que realiza, son fundamentales para guiar el aprendizaje. La planificación y ejecución del currículo compromete en una línea de igual importancia, la forma como el docente guía o estimula el aprendizaje del pensamiento hacia la elaboración de análisis, síntesis, evaluación, reflexión, resolución de situaciones y toma de decisiones (Bloom, 1974). El docente también puede formar personas pasivas, reproductoras de conocimientos, al limitar el potencial estudiantil a la literalidad o al pensamiento concreto.

Se debe valorar la plasticidad cognitiva de los estudiantes durante la infancia para la generación de ideas que pasen por actividades mentales deconstructivas y constructivas. Toda sociedad avanza cuando sus miembros desarrollan una mirada reflexiva ante el lenguaje y el pensamiento. Esto nos da a conocer que los conocimientos, así como se aprenden, también se reaprenden en la dinámica de la enseñanza y aprendizaje.

Además, referido al pensamiento crítico, se fundamenta en el concepto dado por Dewey (1998), para quien el pensamiento es la idea o ideas que la persona tiene. Ahora bien, el pensamiento no es único porque se piensa para idear, para realizar una acción, para organizar una tarea; etc.; pero fundamentalmente se piensa para reflexionar. Es decir, pensar críticamente es reflexionar sobre una idea en correspondencia con otra, siendo la segunda idea base de la que continúa, y esta, una consecuencia de las ideas primigenias. Este proceso cíclico del pensamiento cuando llega a una conclusión, se dice que es reflexivo o crítico, porque genera una nueva idea y motivo para nuevas reflexiones. 
Se dice que pensamos críticamente cuando hacemos de la reflexión una actividad constante, sea al analizar aquello que vemos o conocemos, al emitir una opinión en el marco del conocimiento o al buscar comprobar con objetividad la naturaleza de las cosas en el contexto real. En otras palabras, pensar críticamente es poseer una mentalidad abierta para buscar por voluntad propia, razones o explicaciones de aquello que se observa y se experimenta en la vida (Facione, 2000).

El mundo actual demanda ser reflexivos, críticos y autocríticos por la infinidad de información digital que nos brindan las TIC; y en este contexto virtual, el docente guía para seleccionar, discriminar, deliberar, organizar y procesar la información, puesto que no todo lo que se expone es veraz. Pensar críticamente es importante como leer críticamente (Páez y Rondón, 2014). Entonces, pensar y aprender a pensar, es de alta responsabilidad para la educación (Raths, Jonas, Rothstein y Wassermann, 2005).

En torno a la capacidad para interpretar, esta se apoya en otras como la comparación, relación, deducción, explicación, interpretación, comprobación. En otras palabras, para interpretar se requiere contar con "pruebas" suficientes que den evidencia de las razones que avalan la conclusión a la cual se llega (Raths et al., 2005).

Por tanto, las sociedades requieren aprender a desenvolverse ante un mundo matizado por la verdad y el engaño, por la apariencia y las propiedades de los hechos. Lo aceptable y lo racional son lo que nos permite tomar una postura frente a un hecho y sustentar con argumentos las razones de las decisiones. Las razones son las que otorgan sentido a lo defendible. Actuar razonablemente, implica aprender este accionar (Habermas, 1999).

La evidencia de haber desarrollado el pensamiento crítico se manifiesta cuando se razona frente a una realidad para tener una idea clara sobre ella. La razón lleva a emitir un juicio sobre lo razonado. El juicio es la conclusión de una buena razón (Siegel, 1990). Por tanto, si hay pensamiento crítico entonces hay razonamiento o racionalidad, la cual se basa en principios. Así, ambos conceptos presentan una relación inherente. Según Paul y Elder (2003), el pensamiento crítico se caracteriza por: plantear un problema con base en preguntas específicas, acopiar y evaluar la información en torno al problema, llegar a conclusiones apoyadas en argumentos, consolida el problema con las conclusiones y presentar un informe sobre el problema, las informaciones que comprende, las soluciones pertinentes y las conclusiones.

El objetivo fue evaluar las estrategias didácticas usadas en sesiones de aprendizaje del área de comunicación para desarrollar el pensamiento crítico en estudiantes de educación secundaria de II. EE. de la jurisdicción de Ocros. 


\section{Metodología}

En referencia al enfoque de la investigación, es de tipo mixto (cualitativo-cuantitativo) porque se busca conocer en el contexto educativo, aquellos procesos didácticos que contribuyen al desarrollo del pensamiento crítico en los estudiantes de educación secundaria. A través del método inductivo se conocerá el fenómeno de estudio para llegar a generalizaciones, teniendo presentes los estudios teóricos. El tipo de estudio según el enfoque mixto (cualitativocuantitativo) es estudios de casos y análisis documental para el primero y descriptivo para el segundo (Hernández y Mendoza, 2018). Asimismo, se han establecido cuatro categorías y tres dimensiones que son:

Actividades pedagógicas: aprendizaje cognitivo en contextos motivantes; situaciones desafiantes en correspondencia con los conocimientos previos, susceptibles de comprenderse y analizarse; propuesta de posibles soluciones; toma de decisiones frente a posibles soluciones; uso de conceptos y categorías lingüísticas para argumentar, deducir, interpretar y llegar a conclusiones.

El razonamiento: formulación de proposiciones; análisis de las preposiciones en el contexto del texto; integración de las preposiciones; comprensión del sentido del texto a través de anáforas; comprensión del sentido del texto a través de conectores; análisis de adverbios que aluden el lugar y distribución del texto; conexión de ideas explícitas e implícitas; emisión de respuestas sobre el propósito del texto y su sentido global; y emisión de las conclusiones del texto.

La argumentación: emisión de opiniones o argumentos frente a problemas en un texto de lectura, o sobre asuntos planteados a modo de propuestas o soluciones; justificación de posibles opiniones o argumentos como respuesta a las interrogantes; búsqueda de información para justificar la opinión u argumento; contrastar o analizar los argumentos u opiniones en según el estado de cuestión.

Toma de decisiones: la identificación de hechos que denotan problema; la identificación de medios para la solución; la determinación de medios para la solución; el análisis de los medios de solución; la jerarquización y la selección de tales medios.

La población de estudio comprende a docentes de la especialidad de comunicación y cada uno de ellos, representante de una institución educativa de la jurisdicción de la provincia de Ocros, región Ancash.

Se utilizaron tres instrumentos para la recolección de información. La bitácora o cuaderno de campo, en la cual se consignó información sobre la labor pedagógica de los docentes en dos 
momentos de observación de clase; se realizaron talleres y a través de la técnica del metaplán, se analizó con 20 profesores, 10 de ellos de la especialidad de comunicación y 10 de la especialidad de educación artística, las causas y consecuencias sobre la falta de generar acciones que contribuyan al desarrollo del pensamiento crítico. La sistematización de la información se levantó según la bitácora en un formulario que mide el nivel de desempeño alcanzado por el docente en la observación de su práctica pedagógica.

La información recogida se procedió a la sistematización en tablas de coherencia lógica, para luego analizar las evidencias encontradas y proceder al proceso de triangulación, con el estado del arte, entrevistas a los docentes observados y los expertos en comunicación, para interpretar, analizar y establecer los efectos generados por los hechos encontrados.

Estas categorías se visualizan en la matriz de categorización empleada en la tabla 1.

Tabla 1. Estrategias didácticas para promover el pensamiento crítico, matriz categorial.

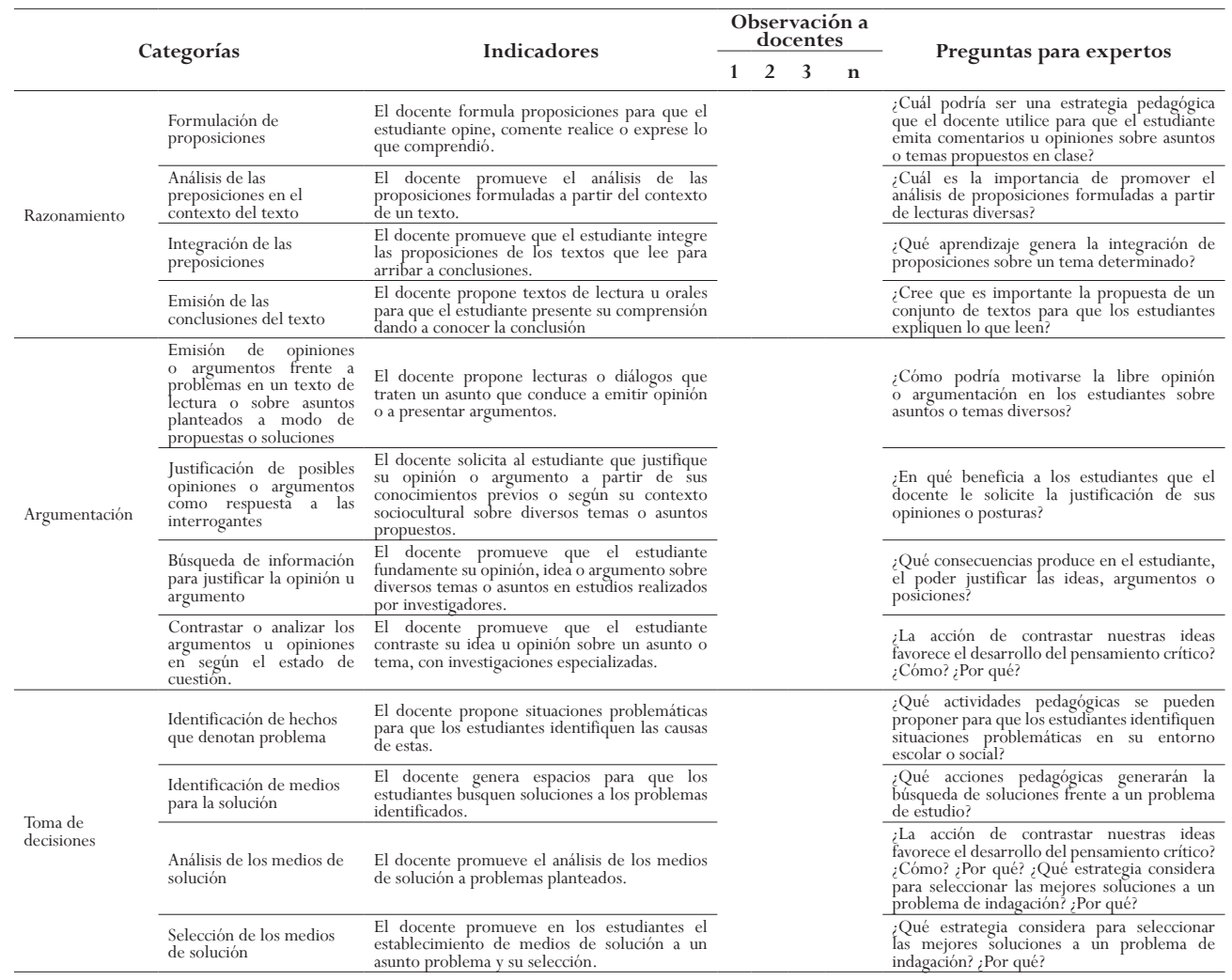


Estrategias didácticas en el desarrollo del pensamiento crítico en estudiantes de educación básica

\section{Resultados}

En encuestas realizadas a los estudiantes de Ocros, referido al desarrollo del pensamiento crítico impulsado y desarrollado por los docentes, el 40,7\% afirman que la gestión del aula es de nivel regular y el 59,3\% es de nivel malo; en la dimensión razonamiento, el 59,3\% afirman que la gestión del aula es de nivel regular y el 40,7\% es de nivel malo; en la dimensión argumentación el 61,9\% afirman que la gestión del aula es de nivel regular y el 38,1\% es de nivel malo; en la dimensión toma de decisiones, el 68,1\% afirman que la gestión del aula es de nivel regular y el $31,9 \%$ es de nivel malo.

Tabla 2. Pensamiento crítico y dimensiones. Percepción estudiantil de la gestión docente.

\begin{tabular}{lcccccccc}
\hline & \multicolumn{2}{c}{$\begin{array}{c}\text { Pensamiento } \\
\text { critico }\end{array}$} & \multicolumn{2}{c}{ Razonamiento } & \multicolumn{2}{c}{ Argumentación } & \multicolumn{2}{c}{$\begin{array}{c}\text { Toma de } \\
\text { decisiones }\end{array}$} \\
\hline & $\mathrm{f}$ & $\%$ & $\mathrm{f}$ & $\%$ & $\mathrm{f}$ & $\%$ & $\mathrm{~F}$ & $\%$ \\
Bueno & 0 & $0,0 \%$ & 0 & $0,0 \%$ & 0 & $0,0 \%$ & 0 & $0,0 \%$ \\
Regular & 46 & $40,7 \%$ & 67 & $59,3 \%$ & 70 & $61,9 \%$ & 77 & $68,1 \%$ \\
Malo & 67 & $59,3 \%$ & 46 & $40,7 \%$ & 43 & $38,1 \%$ & 36 & $31,9 \%$ \\
Total & 113 & $100 \%$ & 113 & $100 \%$ & 113 & $100 \%$ & 113 & $100 \%$ \\
\hline
\end{tabular}

Fuente: elaboración propia.

Al realizarse las observaciones de la gestión del aula a los docentes, se comprueba la percepción de los estudiantes, quienes mayoritariamente perciben la mala gestión en torno al desarrollo del pensamiento crítico, determinándose que durante sus sesiones de aprendizaje y en las diversas subcategorías hay dificultades; así, en relación a la formulación de proposiciones, a través de preguntas, presentando imágenes o explicando situaciones reales que ocurren en su entorno, los estudiantes no son conducidos u orientados por el docente para analizar o evaluar dichas proposiciones o preguntas que no son cuestionadoras, donde generalmente los propios docentes intervienen para absolverlas, no dejando la participación del estudiante.

Así, por ejemplo, el docente Pedro platea las siguientes proposiciones: ¿Qué ven? ¿Cómo se llama? ¿Cómo ha sido la literatura inca? ¿Los antepasados habrán cultivado algún género literario? Estas preguntas quedaron en el vacío, puesto que los estudiantes poco o nada respondieron, en la medida que no se establecieron mecanismos o generaron habilidades para que el estudiante desarrolle el pensamiento crítico.

Con relación al análisis de las preposiciones de contexto, los docentes no presentan interrogantes que los lleven a la reflexión o comprensión de los hechos. Si lo hacen, son los estudiantes que no poseen las herramientas y procesos mentales que permitan el análisis y reflexión. Por eso, responden literalmente a las preguntas desde sus saberes previos, confirmando la no existencia de una enseñanza dirigida para que ellos analicen y sinteticen la información. 
En referencia a la integración de las preposiciones planteadas por los docentes, ellos mismos presentan limitaciones en la integración de las mismas proposiciones planteadas, por lo tanto, es de suponer que los estudiantes no comprenden este proceso de integración de las diferentes proposiciones docentes.

Con referencia a la redacción de las conclusiones del texto, realizadas por los estudiantes, los docentes no guían a los estudiantes sobre las lecturas o explicaciones dadas, demostrando que los estudiantes poseen poco dominio de la temática, con respuestas literales sin reflexión. Las conclusiones sobre los asuntos de aprendizaje, por lo general las aborda el propio docente, excepto, en el caso de dos docentes que al final intentan conducir a los estudiantes en la elaboración de las conclusiones propias con base en los documentos de trabajo que utiliza. Mas, los estudiantes se limitan a escuchar las respuestas a preguntas emitidas por sus docentes.

Podemos afirmar, por tanto, que el componente razonamiento, en las instituciones evaluadas a través de los docentes de comunicación, no desarrollan la competencia referida, debido fundamentalmente a dos factores: el primero debido a las limitadas competencias docentes, pues el desconocimiento o falta de estrategias didácticas no permite promover el desarrollo de los estudiantes, este aspecto es notorio del fracaso y, segundo, la carencia de capacidades en los estudiantes, donde los hábitos de estudios, estrategias de aprendizaje y su falta de deseo por aprender son factores limitantes para su desarrollo. Dos factores complementarios que profundizan la crisis educativa y desarrollo de las competencias en el estudiante.

Esto genera en los estudiantes, e inclusive en los docentes, que los argumentos presenten muchas dificultades, siendo estas en su mayoría literales, no reflejando consistencia y coherencia en el pensamiento en función a los temas.

Por ello, la justificación de los argumentos como respuestas a las interrogantes, los estudiantes responden con adverbios "si" o "no" pero no justifican la afirmación o negación, con lo cual se interpreta como limitado en su pensamiento y expresión. Esto es correlato de la búsqueda de información para la justificación de la opinión y argumento, donde la mayoría de los docentes centra su enseñanza en el tema del libro o cuaderno de trabajo dotado por el Ministerio de Educación, siendo este el único medio para la obtención de información. Por tanto, nos induce a expresar que los estudiantes carecen de herramientas para realizar el pensamiento crítico o indagar en otras fuentes sobre los temas tratados en clase.

Sin otras fuentes del conocimiento, teniendo como medio solo el libro como único elemento no permite acceder a un conocimiento amplio de los bagajes que presentan las diferentes bases de datos y contrastar o analizar los argumentos u opiniones según el estado de cuestión o la teoría científica. Por ello, las preguntas realizadas por los docentes no son absueltas por los estudiantes debido a que son muy ambiguas, poco precisas, ello genera que el propio docente responda a sus propias preguntas generadas. 
Estrategias didácticas en el desarrollo del pensamiento crítico en estudiantes de educación básica

Esto nos lleva a deducir que la identificación de hechos que denotan el problema es complicado, aun cuando los docentes intentan conducir a los estudiantes hacia la identificación de hechos en los temas de las sesiones que desarrollan, pero, solo se quedan en asuntos que genera poca comprensión en los estudiantes por explicaciones poco claras e inconsistentes. Además, los estudiantes no realizan actividades de investigación sobre problemas que afectan el contexto social en el que se desenvuelven o no tienen una participación en la solución de estos.

Por otro lado, existen carencias de estrategias metodológicas que permitan el desarrollo del pensamiento crítico, convirtiéndose la práctica pedagógica en una simple transmisión y reproducción de ideas, hechos aislados que no promueven la reflexión y la propuesta a la solución de los problemas planteados

\section{Discusión}

Los resultados descriptivos y del producto de la observación directa realizada nos muestran los bajos niveles de realización por parte del docente y del estudiante en torno al pensamiento crítico, como también lo presentan las diversas evaluaciones nacionales e internacionales realizadas. En el caso del docente, no está creando las condiciones (medios-materialesestrategias) básicas para este desarrollo como lo afirma Espinoza (2016), sino también los estudiantes carecen de herramientas intelectuales para ejecutar dichos procesos; ambos procesos denotarían una formación integral consistente, y afirmó Morin (1999), coadyuvar a la solución de problemas, sean sociales o de comportamiento de la persona.

Los estudiantes han percibido la mala gestión de los procesos escolares en el aula, y debido a lo que Tobón (2013) y Delors (1996) ya lo señalaban, la carencia en los cuatro niveles de aprendizaje y la incoherencia de las acciones educativas carente de creatividad e innovación, generando desinterés en los estudiantes, olvidando que el principal factor del éxito se basa en los procesos didácticos.

Los procesos didácticos no están generando la reflexión y valoración por parte de los estudiantes, a pesar de la transversalidad en todo acto educativo. Esto lo confirma el experto entrevistado en temas didácticos, Santiago Gallarday, quien afirmó que uno de los problemas es no saber pensar con marcada responsabilidad del docente.

Cuando se analiza la categoría proposiciones de contexto, se ha comprobado que el actuar pedagógico es literal, básico, elemental, que bajo esas condiciones es inviable el desarrollo de las competencias deseadas. Medina (2017) señalaba la necesidad de desarrollar procesos didácticos y un conjunto de estrategias que sugieren para este desarrollo. Esto nos lleva a toma de decisiones pertinentes y adecuadas para el logro de las competencias planificadas. 
Los especialistas entrevistados, Flor Sánchez y Miguel Inga, afirman que en el proceso de la emisión de las conclusiones de un texto o de una situación problémica el estudiante debe tener una mirada holística sobre el asunto. Con este fin, revisará materiales de consulta diversas desde una mirada crítico-reflexiva, evitando el agotamiento de estos. Aquí se debe tener en cuenta que la lectura es el único medio para aprender desde las experiencias vivenciales de otros, que conduzca a pensar con un mejor criterio sobre el asunto en cuestión.

En la segunda categoría, la integración de las proposiciones propuestas para el desarrollo de la sesión, se observan limitaciones de ambos actores, deduciendo que carecen de competencias de este nivel y, además, de conducir el proceso, evidenciando su literalidad de pensamiento. No obstante, la no integración de estas proposiciones no generaría la creatividad mental, el aprendizaje holístico. Elder y Paul (2005) afirmaban que al desarrollar estos aspectos generarían en los estudiantes la autonomía deseada para lograr su aprendizaje. Esto es producto desde la formación inicial del estudiante.

Cuando la persona es capaz de integrar diversas ideas o proposiciones será capaz de llegar a conclusiones, puede haber logrado la reflexión crítica o haber generado una nueva idea. De allí que los expertos concluyan que al lograr esto el estudiante tendrá una mirada holística, crítico-reflexiva, teniendo en cuenta que la lectura es el único medio para aprender desde las experiencias vivenciales de otros que conduzca a pensar con un mejor criterio sobre el asunto en cuestión; sin embargo, lo cuestionable del sistema educativo es la implementación en las escuelas el libro único, la carencia de medios virtuales que imposibilitan a desarrollar estas competencias.

Sabemos que la búsqueda de información para justificar la opinión o el argumento es importante para desarrollar el conocimiento, que se encuentra en la diversidad de fuentes, la variada información, que genera desequilibrio cognitivo respecto a lo que sabe o afirma el estudiante, conduciéndolo a asumir una postura inquieta frente a lo conocido.

Esta acción pedagógica incide en la formación de personas autónomas que pasan de la simple recepción de información al procesamiento de ella. Esta acción pedagógica induce a los estudiantes a explorar e inquirir en el campo de lo poco conocido con interés, buscando permanentemente razones, respuestas o conocimientos que esclarezcan las dudas e inquietudes en el campo del saber que poseen.

La contratación de argumentos u opiniones favorece la capacidad de recepción frente a lo que sabe o conoce; es decir, le otorga la posibilidad de tener una mentalidad abierta para no limitar el concepto o las ideas a una verdad obtusa. Así, comprende que la verdad que postula no es absoluta, Por lo tanto, cuando contrasta la verdad que postula, da lugar al libre pensamiento, la misma que lo conduce a preguntarse constantemente, por la naturaleza de algo, poniendo en 
Estrategias didácticas en el desarrollo del pensamiento crítico en estudiantes de educación básica

cuestionamiento lo que llama verdad. Para ello recurre a preguntas como: ¿por qué es así y no de otra manera? Con esta acción, abre las puertas para el razonamiento y la reflexión en torno a todo lo que tiene un significado.

La forma como debe abordarse la identificación de los hechos que denotan un problema, por los estudiantes en equipos de trabajo, para arribar a una conclusión conjunta, debe partir de sus saberes previos, dando a conocer cómo los problemas les afectan en su vida personal y en la comunidad, pues cada estudiante realizará la búsqueda de soluciones a los problemas que los aqueja. Esta acción despierta interés por la investigación, la ciencia y la cultura. En el proceso el docente realizará preguntas para la identificación de soluciones creativas que innoven los medios de respuesta a los problemas presentados.

\section{Conclusiones}

El desarrollo del pensamiento crítico en la educación peruana y especialmente en la provincia de Ocros-Huaraz, no ha desarrollado como había previsto el órgano rector del país, donde a pesar de los procesos de capacitación e inducción realizada, no ha logrado que los docentes desarrollen metodologías y estrategias que posibiliten que los estudiantes impulsen las competencias y capacidades necesarias para tal cometido.

El análisis realizado, tanto cuantitativo como cualitativamente, arrojan resultados nada halagadores, tanto generales como en las subcategorías, donde docentes y estudiantes no han desarrollado las competencias requeridas, por parte del docente, y presentan limitadas capacidades pedagógicas; así como los estudiantes que no han desarrollado las herramientas cognitivas que les permitan desarrollar el pensamiento crítico.

Estos hallazgos permiten establecer la necesidad de replantear las políticas educativas, reorientando los procesos de la formación continua de los docentes y el diseño curricular escolar. Para el primero de los casos un modelo que priorice las nuevas prácticas pedagógicas, con soporte tecnológicos a través del uso de las TIC, utilizando técnicas como el coaching, profundizando el nivel de conocimiento cognitivo y, para el segundo, que fortalezca las habilidades duras, muy dejadas a menos por la priorización de las habilidades blandas. 


\section{Referencias}

Aguilera, N. y Andueza, A. (2018). Enseñar y aprender la coherencia textual: una propuesta didáctica a partir de la teoría de Michel Charolles. Didáctica, Lengua y literatura, 30. doi:. http: / /dx.doi.org/10.5209/DIDA.61952.

Arias, G. (2018). La lectura crítica como estrategia para el desarrollo del pensamiento lógico. Boletín Virtual, 7(1). Recuperado de https://dialnet.unirioja.es/descarga/ articulo/6297228.pdf.

Avendaño, G. (2016). La lectura crítica en Educación Básica Secundaria y Media: la voz de los docentes. Cuadernos de Lingüística Hispánica, (28), 207-232. doi: http://dx.doi. org/10.19053/0121053X.4916.

Bloom, B. (1974). Taxonomía de los objetivos de la educación. La clasificación de las metas educacionales. Recuperado de https://www.slideshare.net/jorgearturosalcido5/taxonoma-de-losobjetivos-de-la-educacion.

Capinzaiki, S., Santana, I. e Moreira, W. (2016). A competência em informação como um fator relevante para a organização do conhecimento: inter-relação entre padrões belluzzo e as categorias das taxonomias de Bloom na organização do conhecimento. Brazilian Journal of Information Science, 10(3) Recuperado de https: / /search.proquest.com/docview/18660 86738 /54A80E8C192A4306PQ/1 ?accountid=37408.

Castelló, M., Clariana, M., Monereo, C., Palma, M. y Pérez, M. L. (2000). Estrategias de enseñanza y aprendizaje. Formación del profesorado y aplicación en la escuela. Barcelona, España: GRAO.

Chrobak, R. (2017). El aprendizaje significativo para fomentar el pensamiento crítico. Archivos de Ciencias de la Educación, 11(12), e031. doi: http: / / dx.doi.org/10.24215/23468866e031.

Delors, J. (1996). La educación encierra un tesoro. Informe a la UNESCO de la Comisión Internacional sobre la educación para el siglo XXI, . Santillana, Ediciones UNESCO. Recuperado de http: / / www.unesco.org/education/pdf/DELORS_S.PDF

Dewey, J. (1968). La ciencia de la educación. $7^{\mathrm{a}}$ ed. Buenos Aires, Argentina: Losada. Recuperado de https: / /leninpzenteno.files.wordpress.com/2014/02/2-la-ciencia-de-la-educaci_n.pdf.

Dewey, J. (1998). Cómo pensamos. Nueva exposición de la relación entre el pensamiento reflexivo y proceso didáctico. Barcelona, España: Paidós.

Drovnikov, A., Vazieva, A., Khakimova, N., Konyushenko, S., Valeyev, A., Maksimova, E. \& Khairullina, E. (2016). Higher School Teachers training model features. International Review of Management and Marketing, 6(2) Recuperado de https://search.proquest.com/ docview $/ 1771256260$ ? accountid $=37408$. 
Elder, L. (2003). La miniguía hacia el pensamiento crítico para niños. Fundación para el pensamiento crítico. Recuperado de https: / / www.criticalthinking.org/resources/PDF/SP-Teachers_ spanish_all.pdf.

Elder, L. y Paul, R. (2005). Una guía para los educadores en los estándares de competencia para el pensamiento crítico. Estándares, principios, indicadores de desempeño y resultados con una rúbrica maestro en el pensamiento crítico. Fundación para el pensamiento crítico. EU. Recuperado de http://eduteka.icesi.edu.co/pdfdir/EstandaresPensamientoCritico_ESPANHOL.pdf.

Ennis, R. (2011). The Nature of Critical Thinking: An Outline of Critical Thinking Dispositions and Abilities. Recuperado de https://education.illinois.edu/docs/default-source/ faculty-documents / robert-ennis / thenatureofcriticalthinking_51711_000. pdf?sfvrsn=7bb51288_2

Espinoza, E. (2016). La formación docente en los procesos de mediación didáctica. Praxis, 12. doi: https://doi.org/10.21676/23897856.1850.

Facione, P. (2000). The Disposition Toward Critical Thinking: Its Character, Measurement, and Relationship to Critical Thinking Skill. Informal Logic, 20(1), 61-8. doi: https://doi. org/10.22329/il.v20i1.2254.

Feldman, D. (2010). Didáctica general. Buenos Aires, Argentina: Ministerio de Educación de la Nación.

Gregorzewski, M. (2018). Where teachers lear through work and students to learn: an empirically informed report on two examples of aeducational innovations from a German School. Studia Pedagógica, 23(2), 137-157. doi: http://dx.doi.org/10.5817/SP2018-2-8.

Grez, F. (2018). Veo, pienso y me pregunto. El uso de rutinas de pensamiento para promover el pensamiento crítico en las clases de historia a nivel escolar. Praxis Pedagógica, 18(22), 65 84. doi: https://doi.org/10.26620/uniminuto.praxis.18.22.2018.65-84.

Habermas, J. (1999). Teoría de la acción comunicativa, I. Racionalidad de la acción y racionalidad social. Bogotá, Colombia: Santillana. Taurus Humanidades.

Hernández, R. y Mendoza, C. (2018). Metodología de la investigación: Las rutas cuantitativa, cualitativa y mixta. McGraw-Hill Interamericana Editores. México.

Júlia, O. y Formosinho, J. (2018). A formação como pedagogia da relação. Revista da FAEEBA, 27(51), 19-28. doi: http://dx.doi.org/10.21879/faeeba2358-0194.

Kalin, J., Peklaj, C., Pečjak, S., Levpušček, M. \& Zuljan, M. (2017). Elementary and secondary school students' perceptions of teachers' classroom management competencies. CEPS Journal: Center for Educational Policy Studies Journal, 7(4), 37-62. Recuperado de https: / / ojs.cepsj.si/index.php/cepsj/article/view/363. 
Kusumoto, Y. (2018). Enhancing critical thinking through active learning. Language Learning in Higher Education, 8(1), 45-63. doi: http:/ /dx.doi.org/10.1515/cercles-2018-0003.

Lanzat, A., Gil, A., Cacheiro, M. y Pérez, E. (2018). Causas del fracaso escolar: un análisis desde la perspectiva del profesorado y del alumnado. Enseñanza \& Teaching, 36(1), 129 149. doi: http://dx.doi.org/10.14201/et2018361129149.

Maccario, D. (2017). Insegnanti in formazione in university. An experience of didactic innovation.Form@re,17(3),277-290.doi:http://dx.doi.org/10.13128/formare-21268.

Madrid, J. (2018). Desarrollo del pensamiento crítico desde el área de ciencias sociales en la educación básica secundaria. Praxis Pedagógica, 18(22), 49-64. doi: http://dx.doi. org/10.26620/uniminuto.praxis. 18.22.2018.49-64.

Magen-Nagar, N. \& Cohen, L. (2017). Learning strategies as a mediator for motivation and a sense of achievement among student who study in MOOCs. Education and information technologies, 22(3), 1271-1290. doi: http: / / dx.doi.org/10.1007/s10639-016-9492-y.

Medina, M. (2017). Estrategias metodológicas para el desarrollo del pensamiento lógico matemático.Didasc@lia:Didáctica y Educación, IX(1). Recuperado de .

Ministerio de Educación (2003). Ley General de Educación $N^{\circ}$ 28044. Lima. Recuperado de http://www.minedu.gob.pe/p/ley_general_de_educacion_28044.pdf.

Ministerio de Educación (2019). Evaluación de los aprendizajes de los estudiantes en la Educación Básica Regular. Recuperado de https://www.gob.pe/institucion/minedu/normaslegales/259702-025-2019-minedu.

Morin, E. (1999) La Cabeza Bien Puesta: Repensar la reforma, re-formar el pensamiento. Buenos Aires, Argentina: Ediciones Nueva Visión.

Muñoz, L. (2019). La gestión basada en retos: una perspectiva hacia la innovación de la gestión educativa. Revista Electrónica Formación y Calidad Educativa. Recuperado de http://www. refcale.uleam.edu.ec/index.php/refcale/article/view/2820.

Páez, R. y Rondón, G. (2014). La lectura crítica. Propuestas para el aula, derivadas de proyectos de investigación educativa. Recuperado de http://biblioteca.clacso.edu.ar/Colombia/fceunisalle/20170117041131/lecturacr.pdf.

Piaget, J. (1991). Seis estudios de psicología. Barcelona, España: Editorial Labor.

Pimienta, J. (2012). Estrategias de enseñanza-aprendizaje. Docencia universitaria basada en competencias. Mexico D F México: Pearson Educación.

Popescu, G. \& Chisega-Negrila, A. (2017). The Pedagogical Effectiveness of Blended Learning in Higher Education. Doi: http://dx.doi.org/10.12753/2066-026X-17-032. 
Raths, E., Jonas, A., Rothstein, A. y Wassermann, S. (2005). Cómo enseñar a pensar. Teoría y aplicación. Buenos Aires, Argentina: Paidós.

Rodríguez, Y. y Pinto, M. (2018). Modelo de uso de información para la toma de decisiones estratégicas en organizaciones de información. Transformação, 30(1), 51-64. doi: https:// doi.org/10.1590/2318-08892018000100005.

Salica, M. (2018). Caracterización de las habilidades del pensamiento crítico para el desarrollo del conocimiento didáctico del contenido en profesores de Ciencias Naturales. Enseñanza \&Teaching, 36(1), 199-221. doi: http: / /dx.doi.org/10.14201/et2018361199221.

Sanz, R. y Serrano, A. (2017). ¿La educación cambia? Repensando el sentido y finalidad de una escuela para todos. Revista Interuniversitaria, 29 (2). doi: http://dx.doi.org/10.14201/ teoredu292167184.

Siegel, J. (1990). New Work on Critical Thinking: Comments on Frímannsson, Holma and Ritola. Recuperado de www.ojs.statsbiblioteket.dk/index.php/spf.

Siegel, H. (2010). CriticalThinking. In P. Peterson., E. Baker. \& B. McGaw. (Eds.), International Encyclopedia of Education (pp. 141-145). Oxford: Elsevier.

Spoden, C. \& Fricke, K. (2018). Measurement of teachers' reactive, preventive and proactive classroom management skills by student ratings - results from a two-level confirmatory factor analysis. Psychological Test and Assessment Modeling, 60(2), 223-224. Recuperado de https: / / search.proquest.com/docview/2064942664?accountid=37408.

Standish, P. y Thoilliez, B. (2018). El pensamiento crítico en crisis. Una reconsideración pedagógica en tres movimientos. Teoría de la Educación. Revista Interuniversitaria, 30(2), 7-22. doi: http://dx.doi.org/10.14201/teoredu302722.

Suárez, D., Colón, C., Cohen, J. y Colpas, E. (2017). Apropiación de la Redes sociales para la aplicación del método Socrático en el pensamiento crítico. Revista del Instituto de Estudios en Educación Universidad del Norte, 23. doi: https: //doi.org//10.14482/zp.22.5832.

Tobón, S. (2013). Formación integral y competencias. Pensamiento complejo, currículo, didáctica y evaluación. Bogotá, Colombia: ECOE.

Toulmin, S. (2003). Los usos de la argumentación. Traducción de Morras y Pineda. Barcelona. Ed. Península. $1^{a}$ ed. 2007. ISBN 978-84-8307-765-8. Recuperado dehttps://www. libreriaproteo.com/libro/ver/410982-los-usos-de-la-argumentacion.html

Vences, A. \& Antony, M. (2017). Classroom management and metacognition of high school teachers. I-Manager's Journal on School. Educational Technology, 12(4), 24-34. Recuperado de https: / / search.proquest.com/docview/1928950896?accountid=37408.

Vygotski, L. (2009). El desarrollo de los procesos psicológicos superiores. Recuperado de http:// www.terras.edu.ar/biblioteca/6/TA_Vygotzky_Unidad_1.pdf. 